\title{
Ultrasound for Critical Care Physicians: The Martian
}

\author{
Jawad Abukhalaf, MD \\ Michel Boivin, MD
}

Division of Pulmonary, Critical care and Sleep Medicine, University of New Mexico School of Medicine

Albuquerque, NM USA

A 54 year old male with a past medical history significant for granulomatosis with polyangiitis (formerly known as Wegener's granulomatosis) and chronic kidney disease presented with hemoptysis and chest pain.

On presentation, he was found to have a $10 \mathrm{~cm}$ right middle lobe cavitary lesion and was subsequently treated with high dose steroids, antibiotics and antifungals based on bronchoalveolar lavage results. On day 9 of his hospital stay the patient was found to have bilateral lower extremity deep venous thromboses that were treated with intravenous heparin. On day 11 of his stay, the patient started experiencing lower abdominal pain and hypotension. The patient was resuscitated with saline. Bedside ultrasonography was performed (Figure 1).

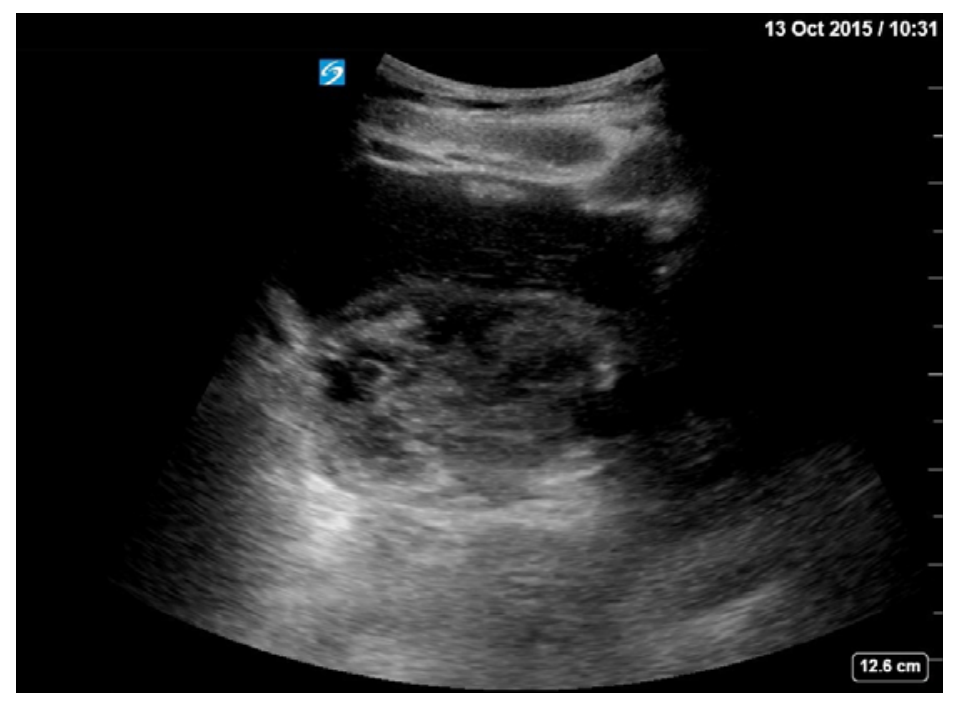

Figure 1. Transverse lower abdominal ultrasound in the pelvis.

What does the transverse view of the lower abdomen (just above the symphysis pubis) demonstrate?

1. Abdominal wall hematoma

2. Distended bladder

3. Horseshoe kidney

4. Psoas hematoma 


\section{Correct! \\ 1. Abdominal wall hematoma}

The patient was diagnosed with an abdominal wall hematoma. The following figures demonstrate the hematoma in CT (Figure 2) and still image of the ultrasound (Figure 3).

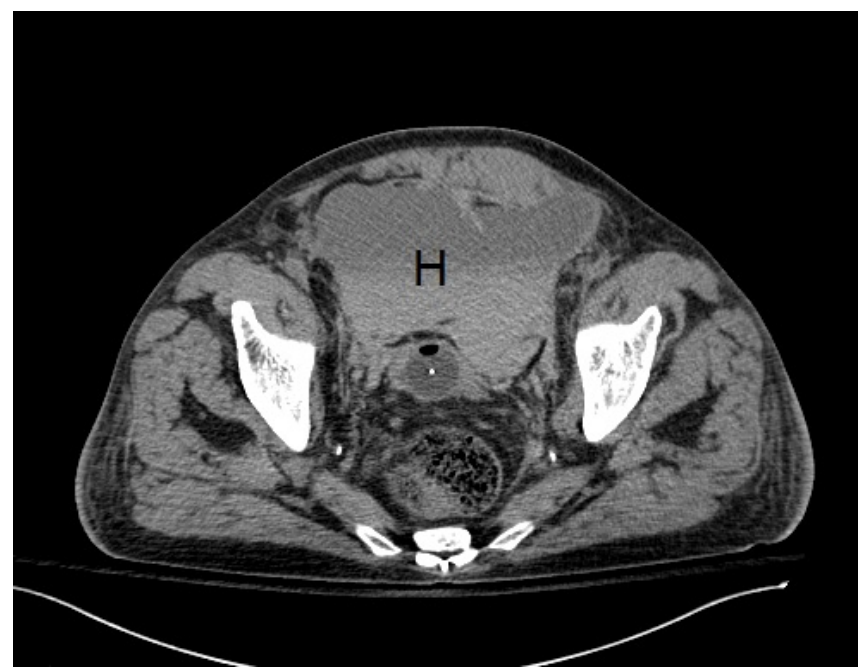

Figure 2. Transverse CT scan view of the pelvis demonstrating abdominal wall hematoma $(\mathrm{H})$.

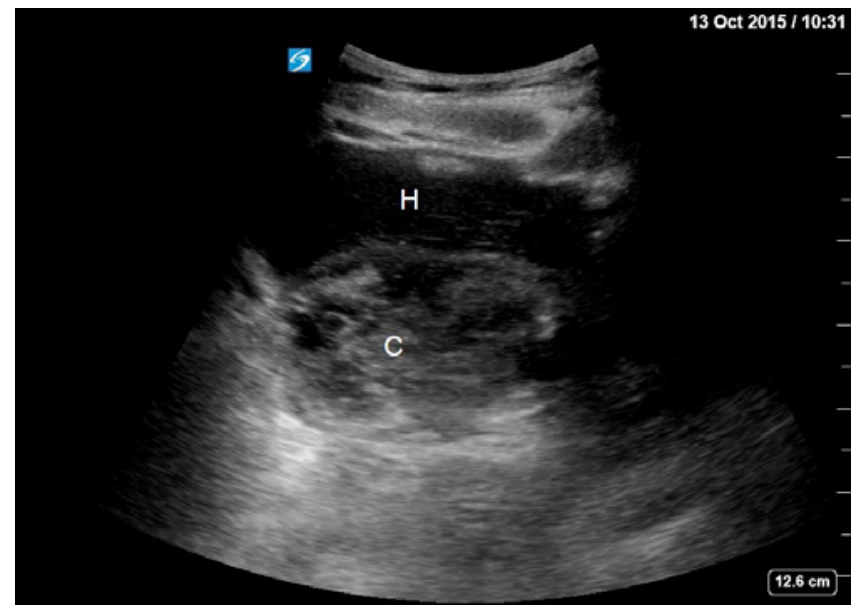

Figure 3. Transverse bedside ultrasound still image of the pelvis with abdominal wall hematoma $(\mathrm{H})$ and clot $(\mathrm{C})$.

Both are labeled with $\mathrm{H}-\mathrm{Hematoma}$. Clot is demonstrated with $\mathrm{C}$ on the ultrasound. Note the improved resolution of ultrasound in demonstrating the texture of the clot. The patient was resuscitated with fluid and blood, the heparin was discontinued, and the shock resolved. 
Hematomas with fresh blood are typically hypoechoic. However, clotted blood may have the appearance of stranding, or organized clots. In larger cavities, bleeding may demonstrate a hematocrit sign due to layering red blood cells after time (1). The hematocrit sign is a gradient of increased, gravity dependent echogenicity seen with layering blood after a hemorrhage within a body cavity.

\section{Reference}

1. Frizzell JD, Griffin LS, Boivin MA, Saeed Al. A 73-year-old man with left lung "white out". Chest. 2015;148(2):e38-41. [CrossRef] [PubMed] 